\title{
Multiple Cross Displacement Amplification Combined with Gold Nanoparticle-Based Lateral Flow Biosensor for Detection of Vibrio parahaemolyticus
}

\author{
Yi Wang ${ }^{1 \dagger}$, Hui $\mathrm{Li}^{1,2 t}$, Dongxun $\mathrm{Li}^{3+}$, Kewei $\mathrm{Li}^{4}$, Yan Wang ${ }^{1}$, Jianguo $\mathrm{Xu}^{1}$ and \\ Changyun $\mathrm{Ye}^{1 *}$
}

1 State Key Laboratory of Infectious Disease Prevention and Control, National Institute for Communicable Disease Control and Prevention, Collaborative Innovation Center for Diagnosis and Treatment of Infectious Diseases, Chinese Center for Disease Control and Prevention, Beijing, China, ${ }^{2}$ Department of Microbiology, Guizhou Medical University, Guiyang, China, ${ }^{3}$ Changping District Center for Disease Control and Prevention, Beijing, China, ${ }^{4}$ Institute of Microbiology, Jilin Provincial Center for Disease Control and Prevention, Changchun, China

\section{OPEN ACCESS}

Edited by:

Learn-Han Lee,

Monash University Malaysia Campus,

Malaysia

Reviewed by: Rolf Dieter Joerger,

University of Delaware, USA

Iddya Karunasagar,

Nitte University, India

${ }^{*}$ Correspondence:

Changyun Ye

yechangyun@icdc.cn

tThese authors have contributed equally to this work.

Specialty section: This article was submitted to

Food Microbiology, a section of the journal

Frontiers in Microbiology

Received: 04 November 2016 Accepted: 06 December 2016 Published: 22 December 2016

Citation:

Wang Y, Li H, Li D, Li K, Wang Y, $X u J$ and Ye C (2016) Multiple Cross Displacement Amplification Combined with Gold Nanoparticle-Based Lateral Flow Biosensor for Detection of Vibrio

parahaemolyticus.

Front. Microbiol. 7:2047. doi: 10.3389/fmicb.2016.02047
Vibrio parahaemolyticus ( $V$. parahaemolyticus) is a marine seafood-borne pathogen causing severe illnesses in humans and aquatic animals. In the present study, multiple cross displacement amplification was combined with a lateral flow biosensor (MCDALFB) to detect the toxR gene of $V$. parahaemolyticus in DNA extracts from pure cultures and spiked oyster homogenates. Amplification was carried out at a constant temperature $\left(62^{\circ} \mathrm{C}\right)$ for only $30 \mathrm{~min}$, and amplification products were directly applied to the biosensor. The entire process, including oyster homogenate processing (30 min), isothermal amplification (30 min) and results indicating ( $2 \mathrm{~min})$, could be completed within $65 \mathrm{~min}$. Amplification product was detectable from as little as $10 \mathrm{fg}$ of pure V. parahaemolyticus DNA and from approximately $4.2 \times 10^{2}$ CFU in $1 \mathrm{~mL}$ of oyster homogenate. No cross-reaction with other Vibrio species and with non-Vibrio species was observed. Therefore, the MCDA-LFB method established in the current report is suitable for the rapid screening of $V$. parahaemolyticus in clinical, food, and environmental samples.

Keywords: Vibro parahaemolyticus, multiple cross displacement amplification, lateral flow biosensor, MCDALFB, limit of detection

\section{INTRODUCTION}

Vibrio parahaemolyticus ( $V$. parahaemolyticus) is a Gram-negative halophilic bacterium that is widely distributed in marine, estuarine, and coastal environments (Letchumanan et al., 2014). The organism is a major food-borne pathogen and frequently isolated from a variety of raw seafoods, such as shellfish, oysters, shrimp, crab, fish and lobster (Wang R. et al., 2015; Letchumanan et al., 2016). In humans, V. parahaemolyticus is able to cause acute gastroenteritis after the consumption of raw, undercooked or mishandled seafood (Su and Liu, 2007). The typical clinical symptoms of $V$. parahaemolyticus infection are abdominal pain and acute dysentery, accompanied by fever, chills, headache, nausea, vomiting, diarrhea, and water-like stools (Shimohata et al., 2010). In rare 
cases, the bacterium is responsible for ear infection, wound infection, or septicaemia that may be life-threatening to populations belonging to special at-risk groups, such as people with immune disorders or liver disease (Centers for Disease Control and Prevention, 2005). In aquatic animals, $V$. parahaemolyticus has the ability to cause serious illnesses in shellfish, fish and penaeid shrimp, resulting in significant losses in aquaculture industries (Tran et al., 2013).

Two hemolysins, thermostable direct hemolysin (TDH) and TDH-related hemolysin (TRH), are major virulence factors for V. parahaemolyticus (Letchumanan et al., 2015). Most $V$. parahaemolyticus strains in seafood samples and environment do not harbor these two hemolysin genes, but virulent strains are often found within larger populations of avirulent strains ( $\mathrm{Su}$ and Liu, 2007; Velazquez-Roman et al., 2012). Since avirulent and virulent isolates have similar growth characteristics, it is difficult to distinguish them phenotypically and therefore, the presence of $V$. parahaemolyticus in general has been as an indicator of seafood contamination.

Traditionally, culture and biochemical methods for identification and detection of $V$. parahaemolyticus from seafood samples can take more than 3 days to complete (Cai et al., 2006). Although PCR-based assays offer more rapid detection, they require instrumentation that might not be readily available in many settings (Levin, 2006; Letchumanan et al., 2014; Law et al., 2015). Multiple cross displacement amplification (MCDA), a novel nucleic acid amplification technique, has been applied in detecting many bacterial agents (Wang Y. et al., 2015; Wang et al., 2016b,c,d). MCDA assay was conducted under isothermal conditions $\left(60-65^{\circ} \mathrm{C}\right)$, thus a simple heater or water bath that maintained a uniform temperature was sufficient. MCDA methods are simple, rapid, highly specific and sensitive, and yield amplifcons from as few as three bacterial cells. Detection of these amplicons can be achieved with disposable lateral flow biosensors (LFB; Wang et al., 2016b,d).

In the current report, a MCDA-LFB assay was established for the rapid detection of $V$. parahaemolyticus strains carrying toxR gene (V. parahaemolyticus-specific gene; Kim et al., 1999). The analytical sensitivity and specificity were determined in pure cultures and in spiked oyster samples.

\section{MATERIALS AND METHODS}

\section{Reagents and Instruments}

Rabbit anti-fluorescein antibody (anti-FITC Ab) and biotinylated bovine serum albumin (biotin-BSA) were purchased from the Abcam Co., Ltd. (Shanghai, China). Streptavidin-immobilized 30-nm gold nanoparticles (SA-G) was purchased from the Resenbio Co., Ltd. (XiAn, China). Membrane backing materials, sample and conjugate pads, nitrocellulose membrane (NC), and absorbent pads were purchased from the Jie Yi Biotechnology Co., Ltd. (Shanghai, China). The Loopamp kits were purchased from Eiken Chemical Co., Ltd. (Beijing, China). QIAamp DNA Mini Kit (QIAamp DNA minikits; Qiagen, Hilden, Germany) was purchased from Qiagen Co., Ltd. (Beijing, China). The visual detection reagent (Hydroxynaphthol blue, HNB) was purchased
TABLE 1 | Bacterial strains used in this study.

\begin{tabular}{|c|c|c|}
\hline Bacteria & Strain no. (source of strains) ${ }^{a}$ & No. of strains \\
\hline \multirow[t]{2}{*}{ Vibrio parahaemolyticus } & ICDC-NVP001 & 1 \\
\hline & Isolated strains & 99 \\
\hline \multirow[t]{2}{*}{ Vibrio vulnificus } & ATCC27562 & 1 \\
\hline & Isolated strains & 4 \\
\hline \multirow[t]{2}{*}{ Vibrio cholerae } & ATCC14035 & 1 \\
\hline & Isolated strains & 4 \\
\hline Vibrio mimicus & Isolated strains & 1 \\
\hline Vibrio fluvialis & Isolated strains & 1 \\
\hline Vibrio alginolyticus & Isolated strains & 1 \\
\hline Plesiomonas shigelloides & Isolated strains & 1 \\
\hline Aeromonas hydrophila & Isolated strains & 1 \\
\hline Enterohemorrhagic E. coli & EDL933 & 1 \\
\hline Enteropathogenic E. coli & Isolated strains & 1 \\
\hline Enterotoxigenic E. coli & Isolated strains & 1 \\
\hline Enteroaggregative E. coli & Isolated strains & 1 \\
\hline Enteroinvasive E. coli & Isolated strains & 1 \\
\hline Shigella dysenteriae & Isolated strains & 1 \\
\hline Shigella boydii & Isolated strains & 1 \\
\hline Shigella flexneri & Isolated strains & 1 \\
\hline Shigella sonnei & Isolated strains & 1 \\
\hline Salmonella & Isolated strains & 1 \\
\hline Enterococcus faecalis & ATCC35667 & 1 \\
\hline Enterococcus faecium & Isolated strains & 1 \\
\hline Listeria monocytogenes & EGD-e & 1 \\
\hline Listeria ivanovii & ATCCBAA-678 & 1 \\
\hline Listeria grayi & ATCC25402 & 1 \\
\hline Listeria innocua & Isolated strains & 1 \\
\hline Listeria welshimeri & Isolated strains & 1 \\
\hline Listeria seeligeri & Isolated strains & 1 \\
\hline Yersinia enterocolitica & ATCC23715 & 1 \\
\hline Enterobacter cloacae & Isolated strains & 1 \\
\hline Bntorobater sakazakii & Isolated strains & 1 \\
\hline Bacillus cereus & Isolated strains & 1 \\
\hline Campylobacter jejuni & ATCC33291 & 1 \\
\hline Pseudomonas aeruginosa & Isolated strains & 1 \\
\hline Staphylococcus aureus & Isolated strains & 1 \\
\hline Staphylococcus epidermidis & Isolated strains & 1 \\
\hline Staphylococcus saprophyticus & Isolated strains & 1 \\
\hline Klebsiella pneumoniae & Isolated strains & 1 \\
\hline
\end{tabular}

aATCC, American Type Culture Collection; ICDC, National Institute for Communicable Disease Control Disease Control and Prevention, Chinese Center for Disease Control and Prevention.

from BeiJing-HaiTaiZhengYuan Technology Co., Ltd. (Beijing, China).

\section{Preparation of Gold Nanoparticle-Based Dipstick Biosensor}

The dry-reagent strips $(4 \mathrm{~mm} \times 60 \mathrm{~mm})$ were prepared as previously described with some modifications (Wang et al., 2016b,d). In brief, the sample pad, conjugate pad, NC membrane and absorbent pad were laminated onto a plastic adhesive backing card. The anti-FITC Ab $(0.15 \mathrm{mg} / \mathrm{ml})$ and biotin-BSA 


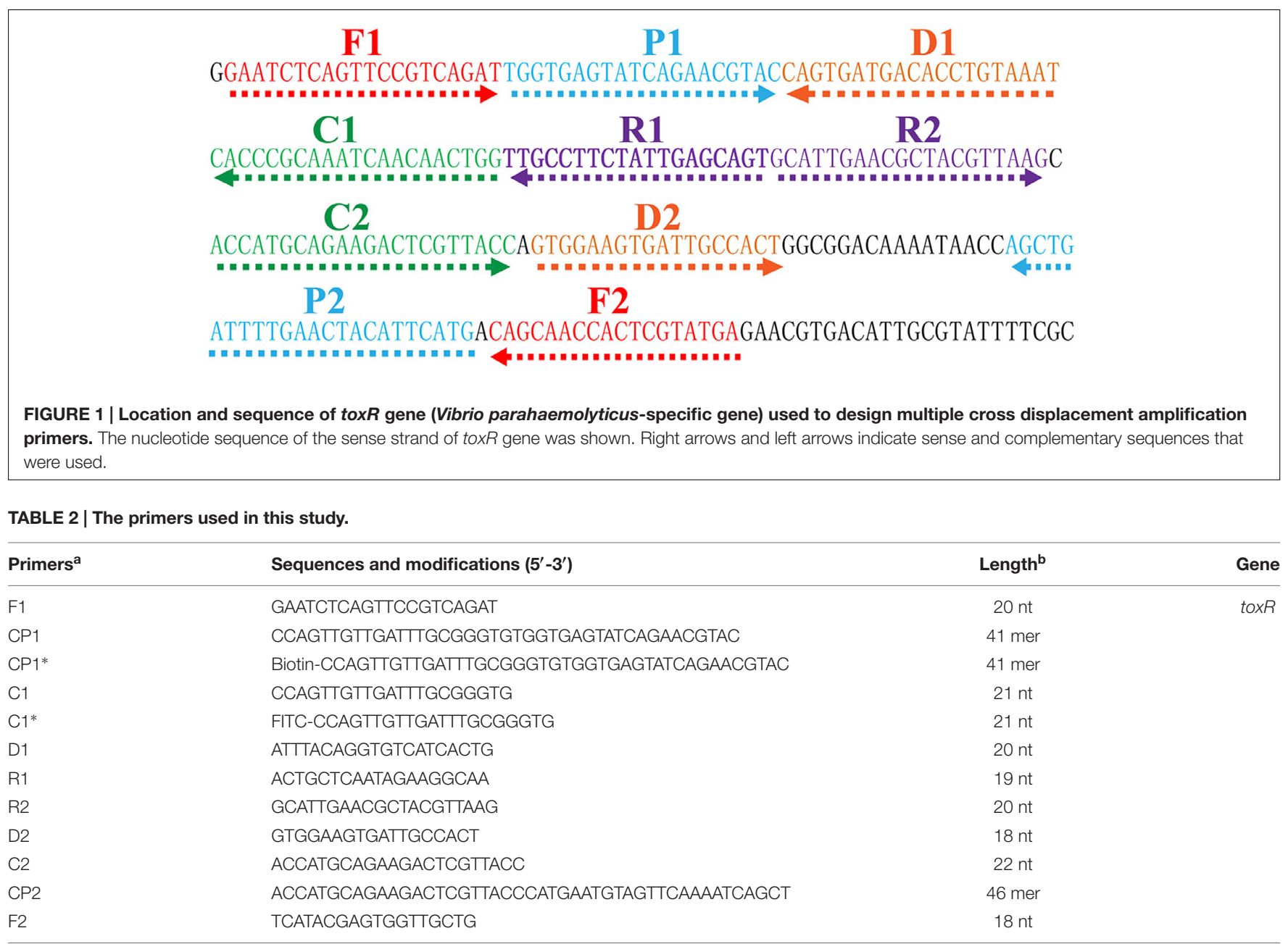

${ }^{\mathrm{a} C P 1}$ *, 5'-labeled with biotin when used in MCDA-LFB assay; $C 1^{*}, 5^{\prime}$-labeled with FITC when used in MCDA-LFB assay. ${ }^{\mathrm{b}}$ nt, nucleotide; mer, monomeric.

$(2.5 \mathrm{mg} / \mathrm{ml})$ conjugates were sprayed onto $\mathrm{NC}$ membrane to form the test line (TL) and control line (CL), with each line separated by $5 \mathrm{~mm}$. SA-G in $0.01 \mathrm{M}$ PBS (PH 7.4) was deposited on the conjugate pad of the strip. The assembled cards were cut into 4 mm wide strips (Deli No. 8012). The assembled biosensors were packaged in a plastic box containing a desiccant gel and stored at the room temperature.

\section{Bacterial Strains and Genomic Template Preparation}

The strains employed in this study (Table 1) were stored in 10\% $(\mathrm{w} / \mathrm{v})$ glycerol broth at $-70^{\circ} \mathrm{C}$. The Vibrio isolates were cultured three times on thiosulfate citrate bile salt sucrose agar (TCBS agar, Eiken Chemical) at $35^{\circ} \mathrm{C}$ and the non-Vibrio strains were cultured three times on nutrient agar plate at $37^{\circ} \mathrm{C}$. Genomic DNA was extracted from all culture strains using the QIAamp DNA Mini Kit according to the manufacturer's instructions and quantified using a Nano drop ND-1000 instrument (Calibre, Beijing, China). V. parahaemolyticus ICDC-NVP001 was serially diluted (10 ng, $10 \mathrm{pg}, 10 \mathrm{fg}, 1 \mathrm{fg}$, and $0.1 \mathrm{fg}$ ) for sensitivity analysis of $V$. parahaemolyticus-MCDA-LFB detection.

\section{Design of MCDA Assay Primers}

The MCDA primer pairs (F1, F2, CP1, CP2, C1, C2, D1, D2, R1 and R2) were designed using PrimerExplorer V4 (Eiken Chemical, Japan) and primer software PRIMER PREMIER 5.0. All primers were analyzed for hairpin structures and hybrids using the Integrated DNA Technologies design tools ${ }^{1}$. Blast analysis was used to verify that the MCDA primers were specific for $V$. parahaemolyticus. The $\mathrm{CP} 1(\mathrm{C} 1+\mathrm{P} 1)$ and $\mathrm{C} 1$ primers were labeled at their $5^{\prime}$ end with biotin and fluorescein isothiocyanate (FITC), respectively. The sequences, positions and modifications of the primer pairs are displayed in Figure 1 and Table 2. All of the oligomers were synthesized and purified by TsingKe Biotech Co., Ltd. (Beijing, China) at HPLC purification grade.

\section{The Standard MCDA Assay}

Multiple cross displacement amplification reactions were carried out in $25 \mu \mathrm{l}$ amplification mixtures as previous studies (Wang Y. et al., 2015; Wang et al., 2016b,c,d). Briefly, each reaction contained $0.4 \mu \mathrm{M}$ each of displacement primers $\mathrm{F} 1$ and $\mathrm{F} 2$, $0.8 \mu \mathrm{M}$ each of amplification primers $\mathrm{C}^{*}$ and $\mathrm{C} 2,1.2 \mu \mathrm{M}$ each

\footnotetext{
${ }^{1}$ http://www.idtdna.com/pages/scitools
} 
A

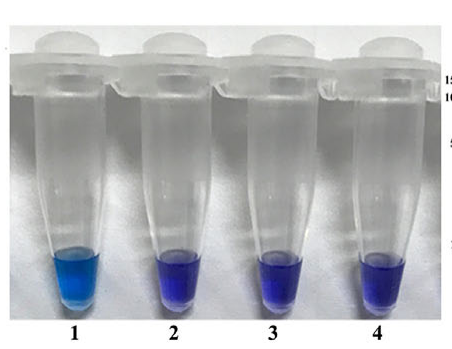

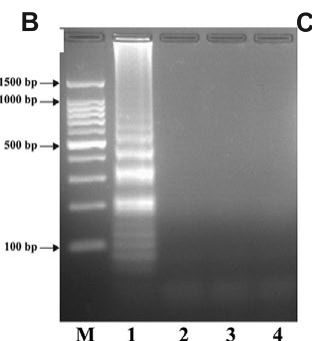

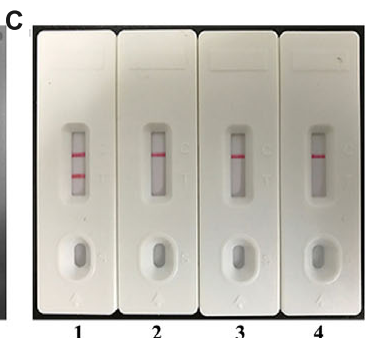

FIGURE 2 | Confirmation and Detection of $\boldsymbol{V}$. parahaemolyticus-MCDA products. (A) By Hydroxynaphthol blue (HNB), amplification products of $V$. parahaemolyticus-MCDA assay were visually analyzed by observation of the color change. (B) Agarose gel electrophoresis of $V$. parahaemolyticus-MCDA products was shown. (C) Lateral flow biosensor applied for visual detection of $V$. parahaemolyticus-MCDA products. Tuble 1/Lane 1/Biosensor 1, positive amplification of $V$. parahaemolyticus strain (ICDC-NVP001); Tuble 2/Lane 2/Biosensor 2, negative control of $E$. faecalis strain (ATCC35667); Tuble 3/Lane 3/Biosensor 3, negative control of S. flexneri strain (ICDC-NPS001); Tuble 4/Lane 4/Biosensor 4, blank control (DW). Lane M, DNA maker DL 100.

A

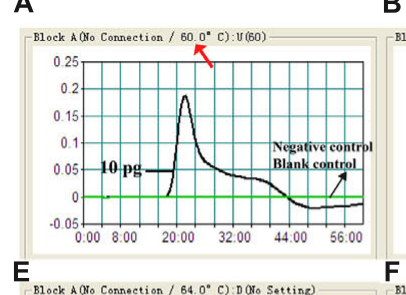

$\mathbf{E}$

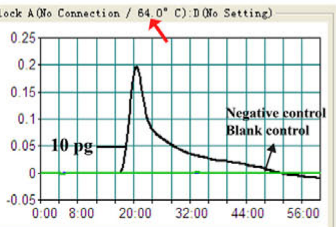

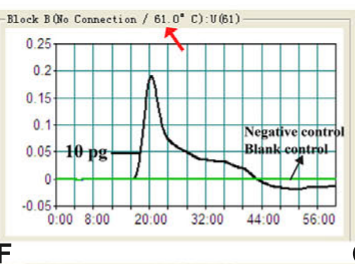

$F_{\text {Bloce }}$

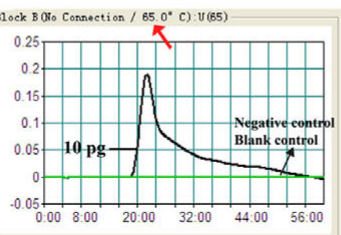

C

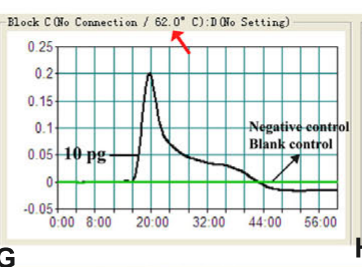

G

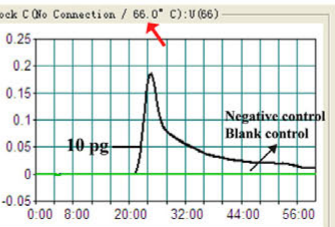

D

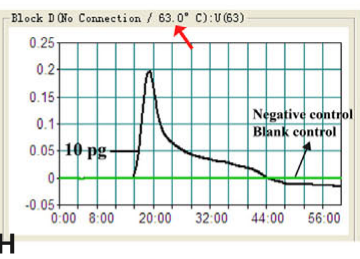

H

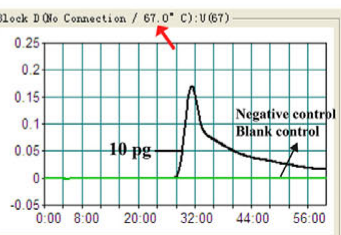

FIGURE 3 | Optimal reaction temperature for $\boldsymbol{V}$. parahaemolyticus-MCDA primer sets. The standard MCDA reactions for detection of $V$. parahaemolyticus were monitored by real-time measurement of turbidity and the corresponding curves of concentrations of DNA were marked in the figures. The threshold value was 0.1 and the turbidity of $>0.1$ was considered to be positive. Eight kinetic graphs $(\mathbf{A}-\mathbf{H})$ were generated at various temperatures $\left(60-67^{\circ} \mathrm{C}, 1^{\circ} \mathrm{C}\right.$ intervals) with target pathogens DNA at the level of $10 \mathrm{pg}$ per reaction. The graphs from B-E showed robust amplification.

of amplification primers R1, R2, D1 and D2, $1.2 \mu \mathrm{M}$ each of cross primers $\mathrm{CP} 1$ and $\mathrm{CP}^{*}, 2.4 \mu \mathrm{M}$ cross primer $\mathrm{CP} 2,12.5 \mu \mathrm{l} 2 \times$ reaction mix (Loopamp DNA amplification Kit), $1.25 \mu \mathrm{l}$ of Bst DNA polymerase (10 U) and $1 \mu \mathrm{l}$ DNA template.

A total of four monitoring methods, including colorimetric indicator (HNB), gel electrophoresis, turbidimeter (LA-320C) and LFB detection, were used for analyzing the amplicons. When employing $\mathrm{HNB}$, the amplified products caused a color change from violet to sky blue, while the negative controls and blank control remained violet. MCDA products were analyzed by $2 \%$ agarose gel electrophoresis, the specific ladder of multiple bands should be seen for positive amplifications, but not in the negative and blank controls. By LFB, two visible red lines (TL; CL) should be observed in positive reactions, and only the control lines were visual in negative and blank controls.

The optimal reaction temperature was determined in the range of 60 to $67^{\circ} \mathrm{C}$ for $60 \mathrm{~min}$. Mixtures with $1 \mu \mathrm{l}$ genomic template of Enterococcus faecalis strains (E. faecalis, ATCC35667) and Shigella flexneri (S. flexneri, ICDC-NPS001) strains were used as negative controls, and mixtures with $1 \mu \mathrm{l}$ double distilled water (DW) were selected as a blank control.

\section{Specificity and Sensitivity of the V. parahaemolyticus-MCDA-LFB Assay}

The specificity of $V$. parahaemolyticus-MCDA-LFB was analyzed with DNA templates from 143 bacterial strains (Table 1). The assays were repeated at least twice. The limit of detection (LoD), which was tested using serial dilutions (10 ng, $10 \mathrm{pg}, 10 \mathrm{fg}, 1 \mathrm{fg}$ and 0.1 fg per microliter), was defined by genomic DNA amount of the template. Detection by $V$. parahaemolyticus-MCDA-LFB was compared to that with a colorimetric indicator (HNB), real time turbidity and $2 \%$ agarose gel electrophoresis. Three replicates of each dilution were tested.

\section{Optimization the Amplification Time of the V. parahaemolyticus-MCDA-LFB Assay}

The optimal time for $V$. parahaemolyticus-MCDA-LFB was determined by increasing the reaction time from 10 to $40 \mathrm{~min}$ at 10 min intervals. The MCDA products were analyzed using LFB detection and two replicates of each amplification time were determined. 


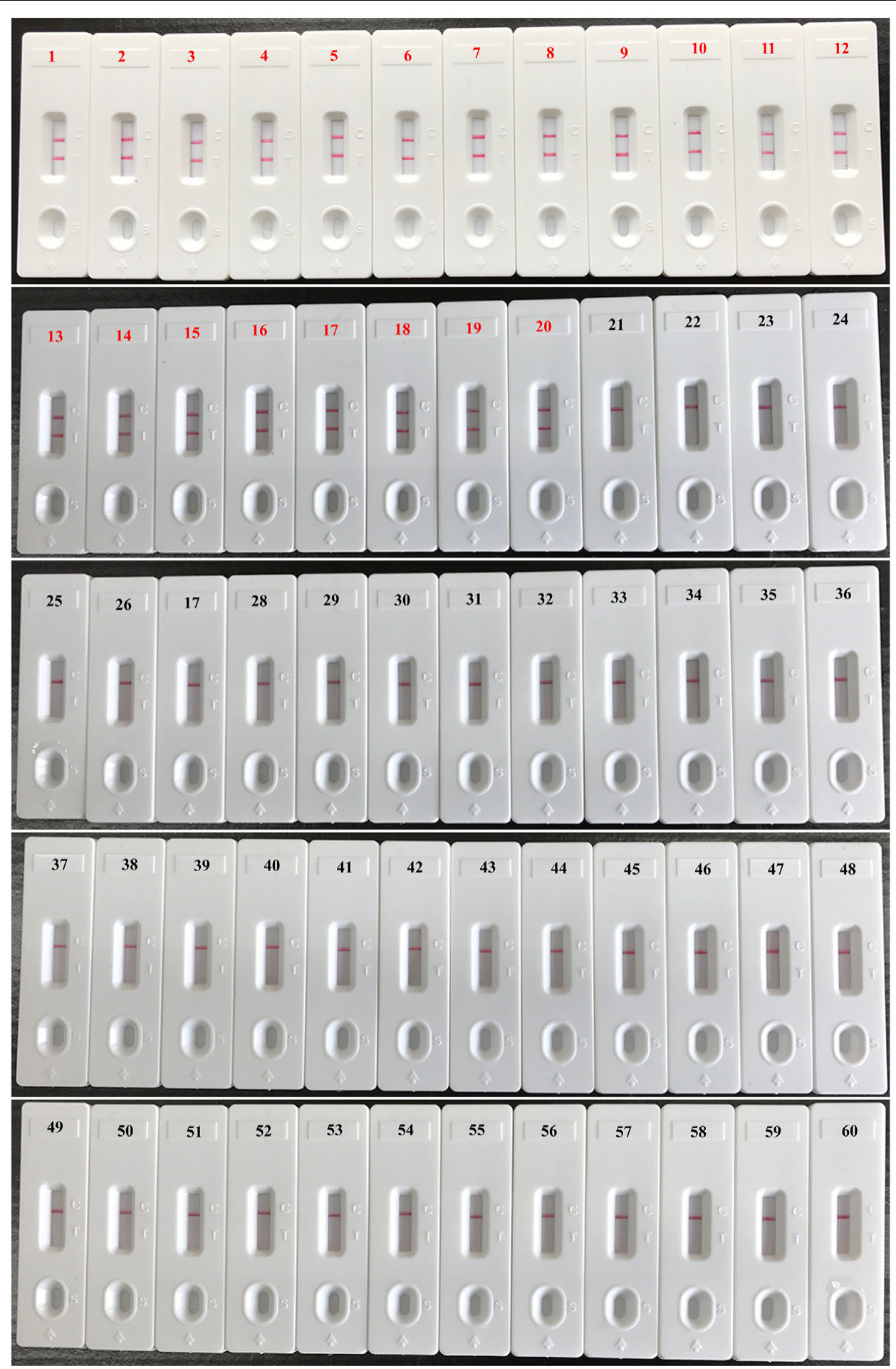

FIGURE 4 | Analytical specificity of $\boldsymbol{V}$. parahaemolyticus-MCDA-LFB assay for different strains. The MCDA reactions were carried out using different genomic DNA templates and were analyzed by means of visual format. Biosensor 1, V. parahaemolyticus strain (ICDC-NVP001); biosensors 2-20, nineteen isolated strains of $V$. parahaemolyticus; biosensor 21, V. Cholerae strain (ATCC14035); biosensors 22-23, two isolated strains of $V$. cholerae; biosensor 24, V. vulnificus (ATCC27562); biosensors 25-26, two isolated strains of $V$. vulnificus; biosensor 27 isolated strain of $V$. mimicus; biosensor 28, isolated strain of $V$. fluvialis; biosensor 29, isolated strain of V. alginolyticus; biosensors 30-59, Plesiomonas shigelloides, Aeromonas hydrophila, Enteropathogenic E. coli, Enterotoxigenic E. coli, Enteroaggregative E. coli, Enteroinvasive E. coli, Enterohemorrhagic E. coli, Shigella dysenteriae, Shigella boydii, Shigella flexneri, Shigella sonnei, Salmonella, Enterococcus faecalis, Enterococcus faecium, Listeria monocytogenes, Listeria ivanovii, Listeria grayi, Listeria innocua, Listeria welshimeri, Listeria seeligeri, Yersinia enterocolitica, Enterobacter cloacae, Bntorobater sakazakii, Bacillus cereus, Campylobacter jejuni, Pseudomonas aeruginosa, Staphylococcus aureus, Staphylococcus epidermidis, Staphylococcus saprophyticus, Klebsiella pneumoniae, biosensor 60, blank control (DW). 
A

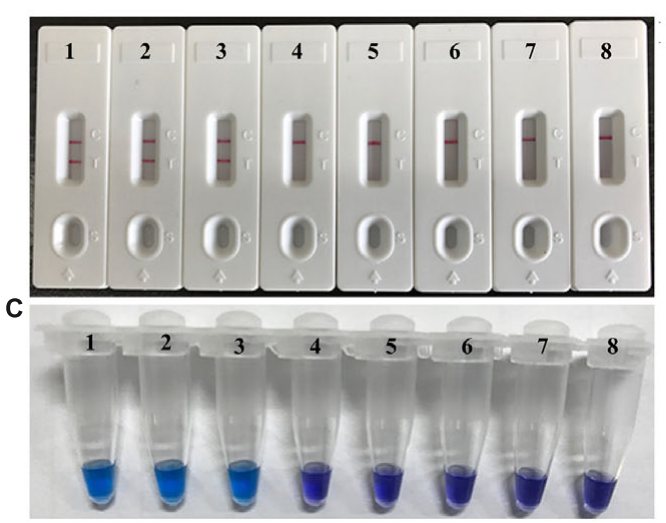

B

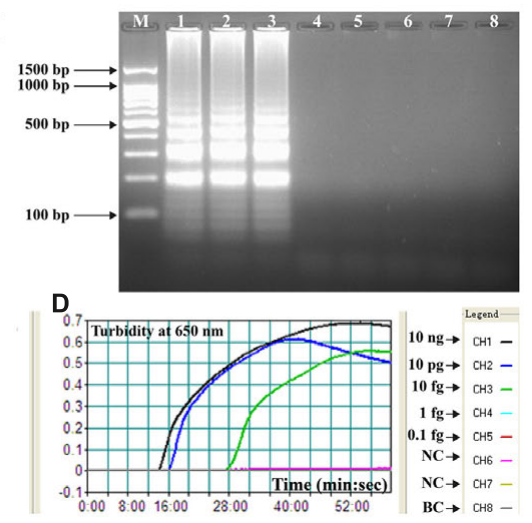

FIGURE 5 | Analytical sensitivity of MCDA-LFB assay using serially diluted genomic DNA with $\boldsymbol{V}$. parahaemolyticus strain ICDC-NVP001. A total of four monitoring techniques, including later flow biosensor (A), gel electrophoresis (B), colorimetric indicator (HNB; C) and real time turbidity (D), were applied for analyzing the amplification products. The serial dilutions (10 ng, $10 \mathrm{pg}, 10 \mathrm{fg}, 1 \mathrm{fg}$, and $0.1 \mathrm{fg}$ ) of target templates were subjected to standard MCDA reactions. Biosensors (A)/ Lanes (B)/Tubes (C)/ Turbidity signals (D) 1-8 represented the DNA levels of $10 \mathrm{ng}, 10 \mathrm{pg}, 10 \mathrm{fg}, 1 \mathrm{fg}$ and $0.1 \mathrm{fg}$ per reaction, negative control (10 pg of $E$. faecalis genomic DNA), negative control (10 pg of $S$. flexneri genomic DNA) and blank control (DW). The genomic DNA levels of $10 \mathrm{ng}, 10 \mathrm{pg}$ and $10 \mathrm{fg}$ per reaction produced the positive reactions.

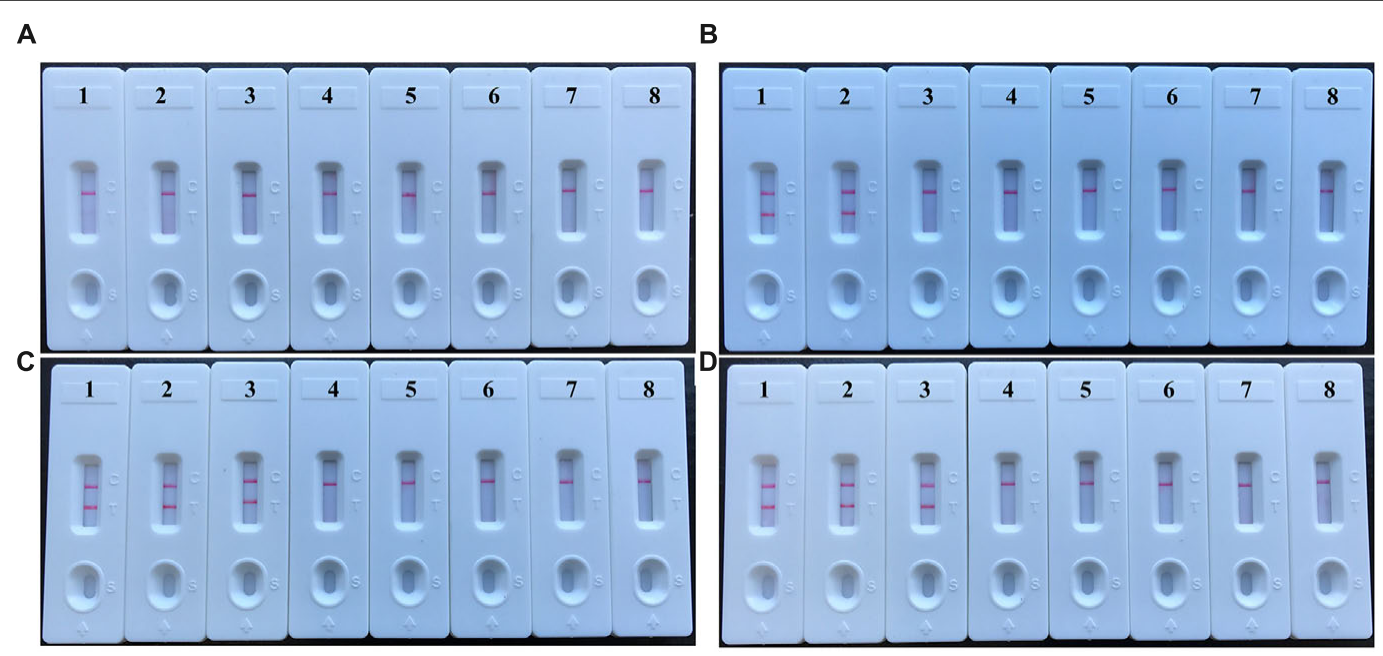

FIGURE 6 | The optimal duration of time required for $\boldsymbol{V}$. parahaemolyticus-MCDA-LFB assay. Four different reaction times (A, 10 min; B, 20 min; C, 30 min; D, $40 \mathrm{~min}$ ) were evaluated and compared at $62^{\circ} \mathrm{C}$. Biosensors 1, 2, 3, 4, 5, 6, 7, and 8 represent DNA levels of $10 \mathrm{ng}$ of $V$. parahaemolyticus templates, $10 \mathrm{pg}$ of $V$. parahaemolyticus templates, $10 \mathrm{fg}$ of $V$. parahaemolyticus templates, $1 \mathrm{fg}$ of $V$. parahaemolyticus templates, $0.1 \mathrm{fg} V$. parahaemolyticus templates per tube, negative control (E. faecalis, $10 \mathrm{pg}$ per reaction), negative control (S. flexneri, $10 \mathrm{pg}$ per reaction) and blank control (DW). The best sensitivity was observed when the amplification lasted for $30 \mathrm{~min}$ (C).

\section{V. parahaemolyticus-MCDA-LFB Detection in Oyster Samples}

Vibrio parahaemolyticus ICDC-NVP001 was added into oyster samples obtained from local seafood restaurants in Beijing. Only oyster samples that tested negative for $V$. parahaemolyticus according to Kim et al. (1999) were spiked. V. parahaemolyticus cultures were serially diluted $\left(10^{-1}\right.$ to $\left.10^{-8}\right)$, and $100-\mu l$ aliquots (appropriate dilution: $10^{-6}$ ) were placed in triplicate on brain heart infusion (BHI). CFUs were counted after $24 \mathrm{~h}$ at $37^{\circ} \mathrm{C}$. Simultaneously, $0.1 \mathrm{~mL}$ of diluted $V$. parahaemolyticus cultures $\left(10^{-3}\right.$ to $\left.10^{8}\right)$ with known amounts $\left(4.2 \times 10^{5}\right.$ to
$4.2 \times 10^{0} \mathrm{CFU} / \mathrm{mL}$ ) was inoculated into $900 \mu \mathrm{l}$ of oyster homogenates and mixed well. The spiked oyster samples were centrifuged at $200 \mathrm{~g}$ for $5 \mathrm{~min}$, and the supernatant was placed into a new tube, and the was centrifuged at $18000 \mathrm{~g}$ for $5 \mathrm{~min}$. The supernatant was removed and the pellet was subjected to extract genomic DNA, and the DNA templates were eluted in $20 \mu \mathrm{l}$ of elution buffer. For the MCDA-LFB assay, $1 \mu \mathrm{l}$ of the extracted DNA was used as template and non-contaminated oyster samples were served as negative control. Three independent assays were performed. 

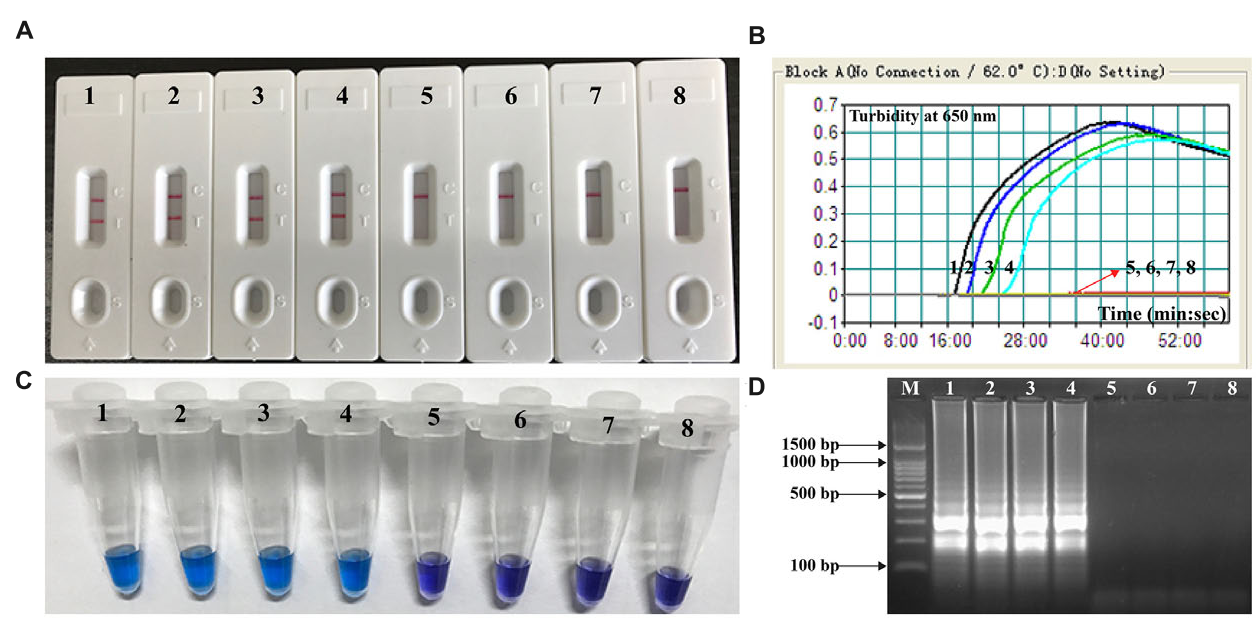

FIGURE 7 | Analytical sensitivity of $\boldsymbol{V}$. parahaemolyticus-MCDA-LFB for detecting target pathogen in oyster samples. Four monitoring techniques, including later flow biosensor (A), real time turbidity (B), colorimetric indicator (HNB; C) and gel electrophoresis (D), were applied for analyzing the amplification products. The serial dilutions of target templates were subjected to standard MCDA reactions. Strips (A)/Turbidity signals (B)/ Tubes (C)/Lanes (D) 1-8 represented the DNA levels of $2100 \mathrm{CFU}, 210 \mathrm{CFU}, 21 \mathrm{CFU}, 2.1 \mathrm{CFU}, 0.21 \mathrm{CFU}$, and 0.021 CFU per reaction, negative control (non-contaminated oyster sample) and blank control (DW). The genomic DNA levels of $2100 \mathrm{CFU}, 210 \mathrm{CFU}, 21 \mathrm{CFU}$, and 2.1 CFU per reaction produced the positive reactions.

\section{RESULTS}

\section{Confirmation and Detection of V. parahaemolyticus MCDA Products}

To determine the availability of $V$. parahaemolyticus-MCDA primers (Table 2), $V$. parahaemolyticus MCDA assays with DNA from pure cultures were carried out at $62^{\circ} \mathrm{C}$ for $1 \mathrm{~h}$. Amplification occurred with DNA from $V$. parahaemolyticus (ICDC-NVP001), but not with E. faecalis (ATCC 35667), S. flexneri (ICDCNPS001) DNA and the DW control (Figure 2). Therefore, the $V$. parahaemolyticus-MCDA primer set was a good candidate for development of the MCDA-LFB assay for $V$. parahaemolyticus detection.

\section{Optimization of the Temperature for V. parahaemolyticus-MCDA-LFB Assay}

To verify the optimum amplification temperature, V. parahaemolyticus strain (ICDC-NPV001) was used as the positive control at the level of $10 \mathrm{pg}$ per reaction and the reactions were monitored by the real time turbidity method. Carrying out MCDA at temperatures from 60 to $67^{\circ} \mathrm{C}$ at $1^{\circ} \mathrm{C}$ increments confirmed that $62^{\circ} \mathrm{C}$ was the most suitable temperature for amplification as indicated by the kinetics graphs displayed in Figure 3. At all tested temperatures, the typical kinetics graphs were generated, with the faster amplification obtained from assay temperatures of $62^{\circ} \mathrm{C}$.

\section{Specificity and Sensitivity for V. parahaemolyticus Detection by MCDA-LFB}

When DNA from the bacteria listed in table 1 was used in MCDALFB assays, only the DNA from the $V$. parahaemolyticus strains provided results. DNA from other Vibrio species and from all
non-Vibrio isolates did not lead to the production of detectable amplification products (Figure 4). Two red lines, including TL and $\mathrm{CL}$, appeared on the strips for the positive tests, and only a red line (CL) appeared on the biosensors, suggesting negative results for non- $V$. parahaemolyticus isolates and blank control.

Serial dilution of the $V$. parahaemolyticus genomic DNA in triplicate and use in MCDA assays demonstrated that as little as $10 \mathrm{fg}$ of template DNA produced sufficient amplified DNA for detection by the four monitoring methods employed (Figure 5). The results obtained using biosensor were in complete accordance with real time turbidity, HNB reagent and agarose gel electrophoresis analysis (Figure 5). Moreover, it was possible to detect the amplicons when the amplification reaction was carried out for 30 min (Figure 6).

\section{Application of MCDA to V. parahaemolyticus-Spiked Oyster Homogenates}

The lowest number of $V$. parahaemolyticus CFUs could be detected in $1 \mathrm{~mL}$ of spiked oyster homogenate was approximately $4.2 \times 10^{2} \mathrm{CFU} / \mathrm{mL}(\sim 2.1 \mathrm{CFU}$ per reaction; Figure 7). The $V$. parahaemolyticus-MCDA assay produced negative results at the concentrations lower than $4.2 \times 10^{1} \mathrm{CFU} / \mathrm{mL}(\sim 0.21 \mathrm{CFU}$ per reaction), negative control and blank control. As seen with MCDA assays utilizing DNA from pure cultures, detection of the amplicons was as sensitive with the LFB method with the other three methods (Figure 7).

\section{DISCUSSION}

The present study demonstrated that multiple cross displacement amplification combined a lateral flow biosensor (MCDA-LFB) utilizing the toxR gene as amplification target is capable of 
detection $V$. parahaemolyticus with excellent specificity and sensitivity. The high level of specificity of the assay is likely due to the utilization of toxR as target gene. Although the sequences of housekeeping genes $(r p o D, r c t B, p y r H, \operatorname{rec} A$, and $g y r B$ ) and $16 \mathrm{~S}$ rRNA gene have also been considered as possible targets, tox $R$ is regarded as gene providing the highest level of discrimination according to phylogenetic tree analysis (Yamamoto and Harayama, 1998; Le Roux et al., 2005; Pascual et al., 2010). Then, the assay's specificity was successfully examined using pure cultures and oyster samples. The test was positive for all $V$. parahaemolyticus isolates, but negative for other Vibrio spp. and non-Vibrio isolates (Figure 4). Hence, the $V$. parahaemolyticus-MCDA-LFB method provided a high degree of selectivity for identifying $V$. parahaemolyticus strains.

In additional to its sufficient specificity, the newly established $V$. parahaemolyticus-MCDA-LFB method was able to detect as little as $10 \mathrm{fg}$ of $V$. parahaemolyticus DNA isolated from a pure culture (Figure 5). The $V$. parahaemolyticus-MCDA-LFB assay was 25 -fold more sensitive than $V$. parahaemolyticus-LAMP method, which only detected $250 \mathrm{fg}$ of template DNA per reaction (Wang et al., 2016a). The detection limit of approximately $4.2 \times 10^{2} \mathrm{CFU}$ in $1 \mathrm{~mL}(\sim 2.1 \mathrm{CFU}$ per reaction) of oyster homogenate was also lower than that of the V. parahaemolyticusLAMP assay (92 CFU per reaction in spiked oyster samples; Figure 7) (Wang et al., 2016a). Although the amplification products could be detected equally with other three methods employed in the current study, LFB is likely the preferred method as reading the results is less subjective and does not require instrumentation.

The $V$. parahaemolyticus-MCDA-LFB assay only required a simple incubation at $62^{\circ} \mathrm{C}$ for $30 \mathrm{~min}$. A variety of portable userfriendly instruments adapted for MCDA reaction exist, the dry block heater (HDT-100C, HengAo, Tianjing, China) being one example. The portable $(18 \mathrm{~cm} \times 22 \mathrm{~cm})$, battery-powered device supports 96 MCDA reactions per assay. The MCDA amplification can be conducted using the commercial isothermal amplification kits (such as Eiken Loopamp kits and NEB Warmstart kits), and an MCDA reaction costs approximately \$3.5 USD. The cost of LFB is estimated to be $\$ 2$ USD per test. Combined with the elimination of labor costs because of the requirements for trained personnel in a certified laboratory, our assay becomes more cost-effective.

The MCDA products were directly analyzed using the biosensor (Figures 2 and 4-7). The entire procedure, including

\section{REFERENCES}

Cai, T., Jiang, L., Yang, C., and Huang, K. (2006). Application of real-time PCR for quantitative detection of Vibrio parahaemolyticus from seafood in eastern China. FEMS Immunol. Med. Microbiol. 46, 180-186. doi: 10.1111/j.1574-695X. 2005.00016.x

Centers for Disease Control and Prevention (2005). Vibrio illnesses after Hurricane Katrina-multiple states, August-September 2005. MMWR Morb. Mortal. Wkly Rep. 54, 928-931.

Kim, Y. B., Okuda, J., Matsumoto, C., Takahashi, N., Hashimoto, S., and Nishibuchi, M. (1999). Identification of Vibrio parahaemolyticus strains at the species level by PCR targeted to the toxR gene. J. Clin. Microbiol. 37, 1173-1177. specimen processing (30 $\mathrm{min})$, isothermal reaction $(30 \mathrm{~min})$ and detection (1 min), could be finished with $65 \mathrm{~min}$. Detection of amplification products with a lateral flow device is not only fast, but also simpler and less error-prone than detection by the other methods employed in the current study (Zhang et al., 2014).

\section{CONCLUSION}

A reliable toxR-MCDA-LFB assay was successfully established for identification of $V$. parahaemolyticus, causing seafood-borne gastroenteritis in human, which could facilitate investigations to detect the etiological agent of food poisoning, surveillance for $V$. parahaemolyticus contamination in seafood, as well as ecological studies related with regions, practices and environmental factors. The MCDA-LFB approach devised here was sensitive, specific and simple, and did not rely on complicated instrument and expensive reagents. The use of lateral flow biosensor could provide an objective, rapid and easily interpretable readout of the method's results. Therefore, the toxRMCDA-LFB method could be regarded as a valuable tool for the rapid screening of $V$. parahaemolyticus isolates in clinical, food and environmental samples, especially, in resource-limited areas of developing countries during epidemic periods.

\section{AUTHOR CONTRIBUTIONS}

YiW, JX, and CY conceived and designed the experiments. YiW, HL, DL, KL, and YaW performed the experiments. YiW, HL, and DL analyzed the data. YiW, HL, DL, KL, and YaW contributed reagents/materials/analysis tools. YiW performed the software. YiW, JX, and CY wrote the paper.

\section{FUNDING}

We acknowledge the financial supports of the grant (Mega Project of Research on the Prevention and Control of HIV/AIDS, Viral Hepatitis Infectious Diseases 2013ZX10004-101 to Changyun Ye) from the Ministry of Science and Technology, People's Republic of China, and grant (2015SKLID507 to Changyun Ye) from State Key Laboratory of Infectious Disease Prevention and Control, China CDC.
Law, J. W., Ab Mutalib, N. S., Chan, K. G., and Lee, L. H. (2015). Rapid methods for the detection of foodborne bacterial pathogens: principles, applications, advantages and limitations. Front. Microbiol. 5:770. doi: 10.3389/fmicb.2014. 00770

Le Roux, F., Goubet, A., Thompson, F., Faury, N., Gay, M., Swings, J., et al. (2005). Vibrio gigantis sp. nov., isolated from the haemolymph of cultured oysters (Crassostrea gigas). Int. J. Syst. Evol. Microbiol. 55, 2251-2255. doi: 10.1099/ijs. 0.63666- 0

Letchumanan, V., Chan, K.-G., and Lee, L.-H. (2014). Vibrio parahaemolyticus: a review on the pathogenesis, prevalence, and advance molecular identification techniques. Front. Microbiol. 5:705. doi: 10.3389/fmicb.2014. 00705 
Letchumanan, V., Ser, H.-L., Chan, K.-G., Goh, B.-H., and Lee, L.-H. (2016). Genome Sequence of Vibrio parahaemolyticus VP103 strain isolated from shrimp in Malaysia. Front. Microbiol. 7:1496. doi: 10.3389/fmicb.2016. 01496

Letchumanan, V., Yin, W.-F., Lee, L.-H., and Chan, K.-G. (2015). Prevalence and antimicrobial susceptibility of Vibrio parahaemolyticus isolated from retail shrimps in Malaysia. Front. Microbiol. 6:33. doi: 10.3389/fmicb.2015. 00033

Levin, R. E. (2006). Vibrio parahaemolyticus, a notably lethal human pathogen derived from seafood: a review of its pathogenicity, characteristics, subspecies characterization, and molecular methods of detection. Food Biotechnol. 20, 93-128. doi: 10.1080/08905430500524275

Pascual, J., Macián, M. C., Arahal, D. R., Garay, E., and Pujalte, M. J. (2010). Multilocus sequence analysis of the central clade of the genus Vibrio by using the $16 \mathrm{~S}$ rRNA, recA, pyrH, rpoD, gyrB, rctB and toxR genes. Int. J. Syst. Evol. Microbiol. 60, 154-165. doi: 10.1099/ijs.0.010702-0

Shimohata, T., Nakano, M., Lian, X., Shigeyama, T., Iba, H., Hamamoto, A., et al. (2010). Vibrio parahaemolyticus infection induces modulation of IL-8 secretion through dual pathway via VP1680 in Caco-2 cells. J. Infect. Dis. 203, 537-544. doi: 10.1093/infdis/jiq070

Su, Y.-C., and Liu, C. (2007). Vibrio parahaemolyticus: a concern of seafood safety. Food Microbiol. 24, 549-558. doi: 10.1016/j.fm.2007.01.005

Tran, L., Nunan, L., Redman, R. M., Mohney, L. L., Pantoja, C. R., Fitzsimmons, K., et al. (2013). Determination of the infectious nature of the agent of acute hepatopancreatic necrosis syndrome affecting penaeid shrimp. Dis. Aquat. Organ. 105, 45-55. doi: 10.3354/dao02621

Velazquez-Roman, J., León-Sicairos, N., Flores-Villaseñor, H., VillafañaRauda, S., and Canizalez-Roman, A. (2012). Association of pandemic Vibrio parahaemolyticus O3: K6 present in the coastal environment of Northwest Mexico with cases of recurrent diarrhea between 2004 and 2010. Appl. Environ. Microbiol. 78, 1794-1803. doi: 10.1128/AEM. 06953-11

Wang, R., Zhong, Y., Gu, X., Yuan, J., Saeed, A. F., and Wang, S. (2015). The pathogenesis, detection, and prevention of Vibrio parahaemolyticus. Front. Microbiol. 6:144. doi: 10.3389/fmicb.2015.00144

Wang, Y., Li, D., Wang, Y., Li, K., and Ye, C. (2016a). Rapid and sensitive detection of Vibrio parahaemolyticus and Vibrio vulnificus by multiple endonuclease restriction real-time loop-mediated isothermal amplification technique. Molecules 21:111. doi: 10.3390/molecules21010111
Wang, Y., Wang, Y., Ma, A. J., Li, D. X., Luo, L. J., Liu, D. X., et al. (2015). Rapid and sensitive isothermal detection of nucleic-acid sequence by multiple cross displacement amplification. Sci. Rep. 5:11902. doi: 10.1038/srep11902

Wang, Y., Wang, Y., Xu, J., and Ye, C. (2016b). Development of multiple cross displacement amplification label-based gold nanoparticles lateral flow biosensor for detection of Shigella spp. Front. Microbiol. 7:1834. doi: 10.3389/ fmicb.2016.01834

Wang, Y., Wang, Y., Zhang, L., Liu, D., Luo, L., Li, H., et al. (2016c). Multiplex, rapid, and sensitive isothermal detection of nucleic-acid sequence by endonuclease restriction-mediated real-time multiple cross displacement amplification. Front. Microbiol. 7:753. doi: 10.3389/fmicb.2016. 00753

Wang, Y., Wang, Y., Zhang, L., Xu, J., and Ye, C. (2016d). Visual and multiplex detection of nucleic acid sequence by multiple cross displacement amplification coupled with gold nanoparticle-based lateral flow biosensor. Sens. Actuators B Chem.

Yamamoto, S., and Harayama, S. (1998). Phylogenetic relationships of Pseudomonas putida strains deduced from the nucleotide sequences of gyrB, rpoD and 16S rRNA genes. Int. J. Syst. Evol. Microbiol. 48, 813-819.

Zhang, X., Lowe, S. B., and Gooding, J. J. (2014). Brief review of monitoring methods for loop-mediated isothermal amplification (LAMP). Biosens. Bioelectron. 61, 491-499. doi: 10.1016/j.bios.2014.05.039

Disclosures: YW and CY have filed for a patent from the State Intellectual Property Office of the People's Republic of China, which covers the novel method and sequences included in this manuscript (Application number CN 201611105333.6).

Conflict of Interest Statement: The authors declare that the research was conducted in the absence of any commercial or financial relationships that could be construed as a potential conflict of interest.

Copyright (c) 2016 Wang, Li, Li, Li, Wang, Xu and Ye. This is an open-access article distributed under the terms of the Creative Commons Attribution License (CC BY). The use, distribution or reproduction in other forums is permitted, provided the original author(s) or licensor are credited and that the original publication in this journal is cited, in accordance with accepted academic practice. No use, distribution or reproduction is permitted which does not comply with these terms. 